\title{
La cooperación técnica española parae refuerzo institucional: elementos parala definición de una estrategia
}

Luis Cámara López"

\section{Introducción}

A proximadamente desde mediados de los años 90 , el refuerzo y modernización institucional, el desarrollo de la capacidad y el buen gobierno vienen ocupando un lugar central en el debate internacional sobre el desarrollo. Reflejo de ello es la presencia reiterada de estos términos en los distintos documentos de política y estrategia, así como su inclusión entre los objetivos básicos de las intervenciones que en distintos sectores se emprenden desde la cooperación bilateral o multilateral para el desarrollo.

Esta tendencia también está presente en la Cooperación Española. En primer lugar, la Ley 23/1998 de 7 de julio de Cooperación Internacional para el Desarrollo, en su artículo 7, dedicado a las prioridades sectoriales, incluye «el fortalecimiento de las estructuras democráticas y de la sociedad civil y el apoyo a las instituciones, especialmente las más próximas al ciudadano» (Agencia Española de Cooperación Internacional 2002, pág. 16). Por su parte, tanto el Plan Director de la Cooperación Española para el periodo 2001-2004 (SECIPI, 2001, pág. 26), como los sucesivos planes anuales y las Comisiones Mixtas de Cooperación bilateral que rigen las intervenciones de cooperación en cada país, establecen asimismo entre sus prioridades sectoriales la participación social, $\mathrm{el}$ desarrollo institucional y el buen gobierno.

Gran parte de las iniciativas españolas en este sector se vienen articulando a través de un amplio abanico de actuaciones de Coope ración Técnica (CT )1, protagonizadas por distintos organismos 0 departamentos de las Administraciones públicas, en ámbitos como la consolidación de la democracia y la protección de los derechos humanos, el desarrollo y reforma legal y judicial, el fortalecimiento de la Administración pública, la gestión financiera y fiscal del sector público, la gestión de procesos electorales, la reforma y modernización de la policía o la descentralización y el fortalecimiento de las administraciones locales en diversos páises en desarrollo - en la actualidad, sólo en laAdministración Central, alrededor de sesenta organismos y departamentos ministeriales organizan regularmente ciclos formativos bajo la modalidad de cursos en España y en el exterior, pasantías, etc., cubriendo un amplio abanico de sectores. Una relación detallada de estos programas puede consultarse en Agencia Española de Cooperación Internacional (2002)- .

Según datos del Comité de Ayuda al D esarrollo (CAD) de la OCDE (M inisterio de Asuntos Exteriores, 2002, página 28), actualmente uno de cada seis proyectos de la Cooperación Española en Iberoamérica están relacionados con el desarrollo institucional, sector al que se estima se destinarán en el periodo 2001-2004 en torno a 19.000 millones de pesetas (114,19 millones de Euros), alrededor del $14 \%$ de la AOD bilateral no reembolsable (SECIPI, 2001, página 26).

Ante la relevancia que el tema viene adquiriendo, el presente artículo constituye un intento de síntesis y reflexión sobre los elementos esenciales a considerar para el diseño de una estrategia sobre refuerzo institucional para la cooperación española.

Se comienza poniendo de manifiesto, por un lado, la necesidad de definir el refuerzo institucional, con el fin de orientar más 
adecuadamente las actuaciones de CT en este ámbito y, por otro, señalando las dificultades que este intento entraña. Asimismo, se abordan los distintos planos objeto de análisis para el diseño de una estrategia de refuerzo institucional y las implicaciones para la CT que, en el caso español, ha estado hasta el momento fuertemente orientada hacia la formación de recursos humanos, sin que haya sido posible analizar objetivamente su contribución al de sarrollo de capacidades en las organizaciones contraparte.

Finalmente, se proporcionan algunas claves para mejorar las posibilidades de incidencia de las acciones de CT en el ámbito del refuerzo institucional2, en línea con el cambio de paradigma que se está produciendo en la cooperación internacional para el desarrollo. Este cambio afecta a la naturaleza y a la práctica de la $C T$, que debe abandonar algunas concepciones, enfoques y prácticas inadecuadas (paquetes estandarizados de CT independientemente de los problemas de capacidad existentes, concepción desde la oferta, excesivo énfasis técnico, etc.) para asumir enfoques más dirigidos a la creación de relaciones de asociación, a la orientación desde la demanda y a la participación y apropiación local, enfoques en los que la cooperación internacional no intentaría hacer cosas para los países en desarrollo sino con ellos. (De velopment Assistance Committee, 1996, página 4).

Entre las buenas prácticas del nuevo modelo de CT propuesto se incluye una mayor profundización en la realización de diagnósticos institucionales de partida, la utilización sistemática de herramientas participativas, la combinación de una gestión por objetivos (en el sentido más amplio del término) con el necesario enfoque de proceso durante la ejecución de estas iniciativas y el reconocimiento de que las intervenciones de cooperación para el refuerzo institucional sólo pueden ofrecer efectos significativos en un plazo largo de tiempo. Todo ello tiene también sus implicaciones en relación con los estilos que deben prevalecer en la CT, en los perfiles del personal que se desempeña profesionalmente en este campo y en las vías para extraer y sistematizar el aprendizaje.

\section{La necesidad de avanzar hacia la definición del fortalecimiento institucional y el desarrollo de capacidades}

La literatura actual sobre la cooperación para el desarrollo, la reforma del sector público y el fortalecimiento de la sociedad civil está plagada de conceptos y términos a menudo utilizados de manera prácticamente intercambiable para designar cosas parecidas, de las que se tiene una idea por lo general meramente intuitiva: ejemplo de al gunos de esos términos son el sdesarrollo de capacidades», el «refuerzo o modernización institucional», la usostenibilidad institucional», la «gobernabilidad», el «desarrollo organizativo», etc.

Comencemos por uno de los conceptos que viene protagonizando el debate sobre el desarrollo y la cooperación en losúltimos años, el concepto de «capacidad» 0 el «desarrollo de la capacidad». Las definiciones, acepciones y matices del mismo son muy variadas y quedaría fuera del alcance y el propósito de este artículo el realizar una exposición pormenorizada de las mismas y de sus implicaciones - LUSTHAUS y otros (1999) y M ENTZ, JCN (1997) ofrecen resúmenes muy ilustrativos de la variedad de enfoques y definiciones de «capacidad» (en http:// www. capacity.org/definit. $\mathrm{html}$ puede ampliarse la información al respecto)- .

Baste indicar que una parte significativa de estas acepciones se concentra en la noción instrumental que identifica la capacidad con las habilidades técnicas y organizativas de individuos, grupos, organizaciones y sistemas más amplios para desarrollar sus funciones de manera eficaz, eficiente y sostenible y fijar y lograr objetivos de desarrollo a lo largo del tiempo.

Otras definiciones (BOLGER, 2000; LAND, 2000) consideran que, además de las habilidades citadas, el concepto de capacidad incorpora otras dimensiones como los valores, relaciones, comportamientos, motivaciones, responsabilidades a nivel social 0 interorganizacional, etc., bien relacionadas con las actitudes individuales o con las características del entorno o sistema en el que individuos y organizaciones se relacionan e interactúan.

La pluralidad de interpretaciones y matices del concepto re vela la vaguedad y generalidad del mismo y su frecuente inutilidad desde un punto de vista tanto práctico como analítico, al poder ser asimilado por su amplitud y contenidos al propio concepto de «desarrollo» (M ORGAN, 1998). Algunos autores alertan asimismo de lo «resbaladizo» de estos términos y del peligro de que se conviertan en un mero eslogan sin un significado conceptual claro (SCHACTER, 2000).

Éste es el riesgo en que se incurre actualmente en el contexto de la cooperación española, en el que idéntico grado de generalidad y confusión rodea al término «refuerzo o fortalecimiento institucional». En efecto, a pesar de su reiterada utilización, incluso como categoría específica de análisis, en la cooperación española no se dispone en la actualidad de una definición operativa del término ni de un método para abordar las intervenciones de refuerzo institucional, si bien éstas se asocian principalmente a cuestiones como la modernización y reforma del sector público 0 a la esfera más amplia de la «gobernabilidad» (esfera bastante desarrollada desde un punto de vista académico pero muy poco desde un punto de vista operativo). 
Si, como se deduce de los principales documentos de orientaciones de la política española de cooperación para el desarro$\| 0$, se pretende que refuerzo institucional constituya un elemento central en sus estrategias de actuación, es absolutamente necesario tratar de dotar al fortalecimiento institucional, a pesar de la dificultad que ello implica, de un contenido y una conceptualización operativa y procedimental que sirva de guía y sistematice las intervenciones de cooperación técnica en este ámbito, ya se trate de programas con objetivos o componentes específicos de refuerzo institucional.

El tema reviste cierta importancia, pues afecta a la misma pertinencia y razón de ser de los estilos y enfoques vigentes en la cooperación técnica en general y en la española en particular. $H$ asta que no se precisen cuestiones básicas como qué es el re fuerzo institucional y en qué consiste, por qué llevar a cabo programas de fortalecimiento institucional, qué objetivos se persiguen a través del refuerzo institucional, a quién 0 a qué hay que fortalecer, a qué nivel y en qué sectores deben desplegarse prefe rentemente las intervenciones de refuerzo institucional, etc., no se podrá hablar con propiedad de las estrategias y de los medios más idóneos para lograrlo 0, al menos, promoverlo.

Ello no impide reconocer que las múltiples dimensiones políticas, sociales, de reparto del poder, etc., propias de este ámbito, condicionan las posibilidades de éxito de nuestros esfuerzos de racionalización y planificación de las intervenciones de re fuerzo institucional, de manera que, con frecuencia, estaremos necesariamente sujetos a altas dosis de incertidumbre acerca de su idoneidad y de su utilidad para lograr cambios significativos.

En todo caso, la indefinición de los atributos del fortalecimiento institucional y el disponer de una idea más intuitiva que conceptual de su significado da lugar a distorsiones importantes en la CT, en varias vertientes:

a) Obstaculiza la fijación de resultados y objetivos verificables (en el sentido clásico del término, es decir, a través de indicadores directos, cuantitativos, independientes en cada uno de los niveles, etc.) en las intervenciones en este ámbito. Q uienes se dedican a tareas de planificación del desarrollo conocen bien la tendencia a establecer objetivos en ocasiones vagos, imprecisos y con cierta ambigüedad en los programas y proyectos de desarrollo, como modo deliberado de facilitar la construcción de consenso en torno a esos objetivos entre los distintos actores involucrados (Ron d IN ELLI,1993).

Por la propia generalidad del concepto y por el contexto de incertidumbre en el que se desenvuelven los programas de refuerzo institucional, esta tendencia es aún más frecuente en dichos programas, lo que a menudo dificulta la identificación de niveles de intervención y la selección de las estrategias más adecuadas en cada caso.
Por otro lado, hay que tener en cuenta la pluralidad de objetivos que cumplen las Administraciones públicas, al gunos incluso contrapuestos3, así como tener presente la posibilidad de la existencia de objetivos no explícitos o espurios. La indefinición puede, asimismo, dar lugar a equívocos y/0 expectativas diferentes entre los implicados acerca de lo que se pretende con este tipo de intervenciones.

b) Teniendo en cuenta que la definición de resultados y objetivos y la selección de indicadores son dos procesos que se alimentan mutuamente, en el reverso de la misma moneda nos encontramos con limitaciones para traducir esos objetivos en indicadores de refuerzo institucional que fijen su nivel de exigencia4. Cuando la selección y asignación de indicadores clásicos entraña dificultades insalvables o es necesario recurrir a múltiples indicadores indirectos para aproximarnos a la naturaleza del objetivo en cuestión, lo normal es que surjan dudas más que razonables acerca de si realmente ese objetivo tiene un significado preciso.

c) La vaguedad del concepto permite que prácticamente cualquier intervención de cooperación técnica, aun en su vertiente más clásica, como la que representan los programas de formación de recursos humanos o la prestación de asistencia técnica, sobre todo en el ámbito del sector público, pueda ser considerada per se como intervención orientada al refuerzo institucional.

La adopción de este enfoque, relativamente común en la cooperación española, supondría admitir que en la propia esencia o natural eza de ese tipo de intervenciones de cooperación técnica, con independencia de que las mismas se encuentren en mayor o menor grado acompañadas de otras acciones 0 integradas en estrategias pertinentes para ese fin, está contenida la generación automática de efectos positivos sobre el fortalecimiento institucional en los países en desarrollo, algo cuando menos discutible, como se verá más adelante.

Uno de los efectos indeseados que esto genera es legitimar, exclusivamente por su supuesta y pretendida contribución cuasi automática al «fortalecimiento institucional», la ejecución de un amplio abanico de intervenciones de CT, algunas de cuales pueden incluso carecer de los mínimos criterios de pertinencia o responder exclusivamente a los intereses corporativos o la iniciativa unilateral de las entidades que las promueven, de manera en ocasiones inercial y desprovista de la necesaria reflexión previa sobre su contribución efectiva al desarrollo.

La otra consecuencia perversa inmediata es que se ofrece, por acumulación de actuaciones de cooperación técnica en el capítulo de refuerzo institucional, la falsa impresión de que en la cooperación española existe realmente una estrategia sistemática y coherente en este ámbito. 


\section{Hacia un modelo para la sistematización de las iniciativas de cooperación técnica para el refuerzo institucional y el desarrollo de capacidades}

U rge pues avanzar hacia la definición de un marco conceptual y un modelo que permitan dotar de cierto grado de sistematización y de operatividad a las estrategias de refuerzo institucional y a las iniciativas que en este ámbito se emprendan en el marco de la cooperación española.

Para la construcción de ese modelo es preciso:

1. Tratar de definir en qué consiste el fortalecimiento institucional y analizar su relación con la CT.

2. Distinguir los diferentes planos en los que las iniciativas de cooperación técnica que persigan el refuerzo institucional y el desarrollo de la capacidad pueden desplegarse.

3. Llevar en la medida de lo posible a términos operativos la definición de capacidad y desarrollo institucional (cuales son las principales habilidades técnicas y organizativas de individuos, grupos, organizaciones e instituciones que es necesario fortalecer en cada caso y en cada contexto).

4. Introducir el debate sobre los mejores medios para conseguirlo, lo que conlleva una reflexión sobre las limitaciones de los enfoques actuales de la CT y la presentación de enfoques alternativos y buenas prácticas.

En las próximas líneas se abordan estas cuatro cuestiones, para lo cual se toma como hilo conductor los principales temas objeto de análisis en la literatura actual de cooperación para el desarrollo institucional - de corte marcadamente funcionalista- y se introducen reflexiones ad hoc sobre la realidad de la cooperación española en este ámbito.

\section{1. ¿Q uées el desarrollo institucional ?}

A la cuestión de la indefinición del término «desarrollo institucional» a la que ya nos hemos referido viene a sumarse, por otra parte, la del debate en torno a su carácter instrumental o finalista, es decir, sobre si se trata de un medio para el logro de objetivos superiores, como por ejemplo la reducción de la pobreza, o la consolidación democrática, o si más bien debe ser contemplado como un objetivo en sí mismo de las políticas de desarrollo.

El Comité de Ayuda al D esarrollo de la OCDE, en su más reciente «Examen en materia de Cooperación para el $D$ esarrollo» dedicado a España, se refiere a ello dejando clara su posición al respecto, cuando afirma «El desarrollo institucional no debe convertirse en un fin en sí mismo, dejando de aportar beneficios a los ciudadanos, a los que se supone que debe servir en última instancia el sector público» (M inisterio de Asuntos Exteriores 2002, página 29). La propia Ley de Cooperación pone el énfasis en las instituciones «especialmente más próximas al ciudadano».

En contraposición, desde otras perspectivas la consolidación de la institucionalidad en entornos donde ésta es débil y la generación de confianza de los ciudadanos ante las instituciones, son considerados como atributos básicos del desarrollo, lo que desde este punto de vista llevaría a admitir el desarrollo institucional casi como un fin en sí mismo.

Al igual que sucede con el término «capacidad», la literatura ofrece diversas concepciones del desarrollo o fortalecimiento institucional.

El Comité de Ayuda al D esarrollo de la OCDE defineel impacto en el desarrollo institucional como la umedida en que una intervención mejora o debilita la capacidad de un país o región de hacer uso más eficiente, equitativo y sosteni ble de sus recursos humanos, financieros y naturales, por ejemplo a través de: a) mecanismos institucionales mejor definidos, más estables, transparentes y aplicados de manera eficaz y previsibley/o b) mejor ajuste de la misión y la capacidad de una organización con su mandato, que se deriva de estos mecanismosinstitucionales) (CAD, 2002, página 25).

Lust HaUs (1996, página 1) sugiere que el fortalecimiento institucional consiste en «ayudar a crear instituciones y organizaciones en el mundo en desarrollo que sean capaces de adaptarse constantementeal entorno con el fin de atraer los recursos (humanosy financieros) requeridos para llevar a cabo su mandato».

Siguiendo a N ORAD, el desarrollo institucional ha de concebirse como un «proceso por el cual losindividuos, las organizaciones y los sis temas sociales aumentan sus capacidades y su desempeño en relación a losobjetivos, recursosy el entorno» (N ORAD, 2000, página 5).

De estas definiciones interesa rescatar varios aspectos inherentes al concepto de refuerzo institucional:

- La idea de proceso que implica, por una parte, resaltar la dimensión temporal del desarrollo institucional y su carácter instrumental para lograr un fin superior. Ambas cuestiones tienen profundas implicaciones en los enfoques a adoptar en las estrategias de CT en este ámbito.

- El aumento o mejora de capacidades (para gestionar recursos, fijar objetivos, llevar a cabo su mandato, etc.) 
como propósito del desarrollo institucional. D esde este punto de vista, que responde más a un modelo de planificación por objetivos que a estrategias basadas en una suma de actividades diversas sin una previa reflexión sobre su finalidad, cabría entender el desarrollo institucional como la utilización de un conjunto de estrategias, enfoques, métodos, actividades y recursos orientados al desarrollo de ciertas capacidades.

- El hecho de que el desarrollo institucional es multidimensional, implicando actuaciones no sólo en el nivel de los individuos, sino también en el de las organizaciones, instituciones y los sistemas sociales de los que forman parte.

- La importancia del entorno, como factor que condiciona las posibilidades de refuerzo institucional y como elemento al que, en última instancia, se aspira a transformar.

\subsection{Los «planos» o niveles del desarrollo institucional}

Para poder establecer con nitidez los distintos planos del desarrollo institucional, resulta imprescindible tomar como punto de partida una diferencia conceptual importante, la que existe entre instituciones y organizaciones.

Las instituciones son convenciones que estructuran el comportamiento humano, pudiendo ser de carácter formal (normas, leyes, constituciones, etc.) 0 informal (pautas de comportamiento, convicciones, valores, principios, códigos de conducta, tradiciones, mercados, etc.). En conjunto, las instituciones definirían las reglas del juego en una sociedad, en la que las organizaciones formales actuarían como jugadores, desempeñando sus funciones y desarrollándose (M O RGAN, 1998, página 4).

Una vez hecha la precisión conceptual, la literatura sobre el tema distingue varios planos o niveles básicos relacionados entre sí - que reflejan otras tantas dimensiones de la capacidad- , en los que las iniciativas de cooperación técnica para el fortalecimiento institucional pueden (y deben) desplegarse6:

a) Plano individual, que incorpora a los actores individuales (personal administrativo y técnico, directivos, planificadores, funcionarios, empresarios, productores, etc.).

b) Plano organizacional, en el que se situarían los organismos o entidades (ya se trate de gobiernos, administraciones públicas, empresas, organizaciones de la sociedad civil, etc.).

c) Plano sectorial, que incluye las políticas en un determinado sector y las relaciones (de coordinación, competencia, etc.) entre las entidades que lo integran.

d) Plano de sistema (nivel institucional o del entorno), que representa el contexto más amplio en el que el proceso de

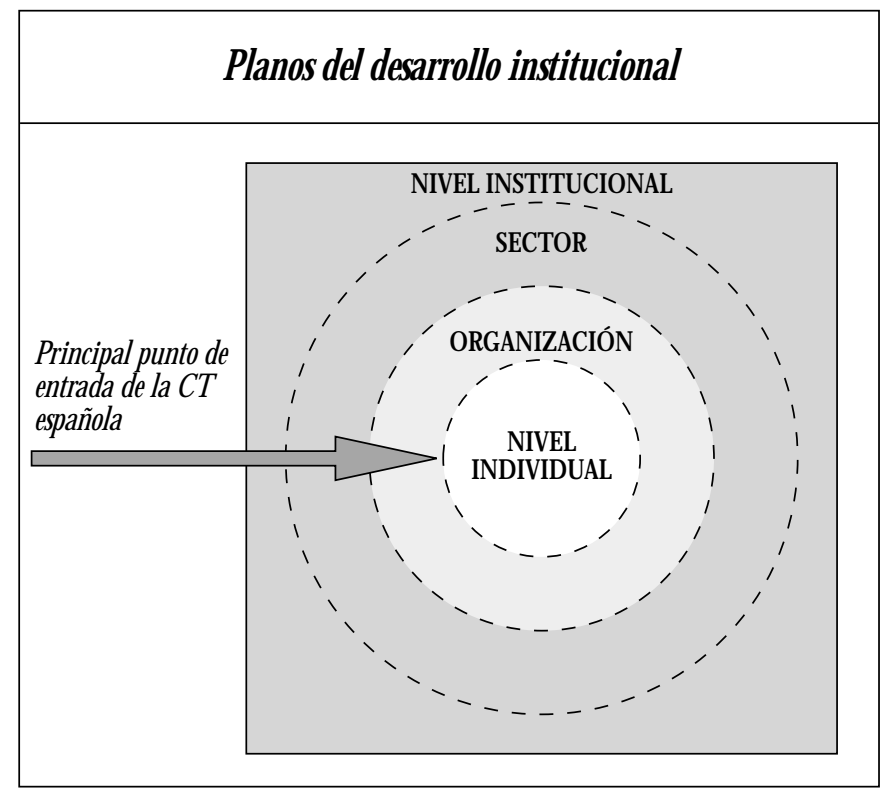

Fuente: N ORAD (2000) y BOLgER (2000).

desarrollo tiene lugar (intuitivamente, al menos, cabría identificar este nivel con la «gobernabilidad»).

Muchas actuaciones de la cooperación técnica española en el ámbito del refuerzo institucional vienen desplegando sus esfuerzos principalmente en el nivel individual, a través de programas diversos de formación de recursos humanos o de asistencia técnica destinados a aumentar las capacidades de individuos en diversos terrenos. En el Catálogo de CT recientemente publicado por $\mathrm{AECI}$ se puede constatar el gran peso que la formación de recursos humanos tiene en las diversas iniciativas incluidas; a éstas habría que añadir los diversos programas de becas, lectorados e intercambios del Ministerio de Asuntos Exteriores de España o la amplia y diversa agenda de programas desarrollados por los $C$ entros I beroamericanos de Formación.

Admitamos que, en última instancia, el funcionamiento adecuado de las organizaciones descansa en un conjunto de capacidades individuales. No obstante, aun reconociendo la importancia de que la CT contribuya a la formación de capital humano de los países en desarrollo, interesa destacar que la actuación intensiva o preferente de la CT en el nivel individual no necesariamente garantiza por sí sola efectos positivos desde un punto de vista del desarrollo institucional, si éstas no están referidas a objetivos correspondientes a los niveles superiores.

Algunas limitaciones evidentes con las que se enfrenta la CT para que se produzca ese salto en un buen número de casos es la alta rotación de personal y la ufuga de cerebros», la ausencia o cuando menos debilidad de la carrera administrativa en la función pública?, el clientelismo que distorsiona los mecanismos de selección y promoción del personal, o sencillamente la falta de incentivos, etc. 
La cuestión relevante que deben plantearse los gestores de CT es, por lo tanto: ¿cómo trasladar o convertir las capacidades individuales en capacidades organizativas?, cuestión que nos conduce al tema de los incentivos para el cambio individual y organizativo en el seno de cada entidad considerada.

En el nivel organizacional se dirimen, entre otros, asuntos re lativos a la estrategia, la planificación y la gestión técnica y financiera, el manejo de los recursos, la estructura organizativa, los procedimientos, la cultura corporativa, la calidad, el liderazgo, etc.

Éste ha sido y es un punto clásico de entrada para las cooperaciones bilaterales de diversos donantes 8 , a través de la prestación de asistencia técnica, equipamiento, infraestructura o apoyo presupuestario. Pero, análogamente, las intervenciones en este plano han de tener presente la referencia al nivel inmediatamente superior, ya se considere el sector en el que las organizaciones se desempeñan o el estrato más amplio referido al sistema político, económico, social e institucional.

Éste es un aspecto que los donantes deben enfatizar en sus relaciones de asociación con las organizaciones de los países en desarrollo, ya que con frecuencia se puede perder de vista que la justificación última de contribuir a la mejora de la capacidad de individuos y a la creación de organizaciones más eficaces, eficientes y sostenibles está en que esas organizaciones presten mejores servicios a los ciudadanos. Ello implica en todo momento tener presentes como referencia última los intereses y prioridades de los usuarios de los servicios y no exclusivamente los de la organización en cuestión. EI CAD, en su examen de la Cooperación Española, se ocupa de esta cuestión indicando que la asistencia a la creación de instituciones debería demostrar su impacto en la población y particularmente en los pobres (M inisterio de Asuntos Exteriores, 2002, pág. 27).

En línea con este argumento, algunos autores señalan que poco puede hacer la CT centrada en este nivel mientras exista el Ilamado «círculo vicioso de la reforma del Estado»9, dado que ésta, aunque involucra aspectos técnicos, es un proceso esencialmente político condicionado por las conductas individuales y las circunstancias locales, y que los incentivos para la transformación de las organizaciones del Estado han de venir básicamente motivados por la presión de la ciudadanía, a la que la iniciativa de la CT no puede sustituir (SCHACTER, 2001). De ahí la importancia de incidir paralelamente, de acuerdo con lo que establecen los distintos documentos de estrategia de la Cooperación Española, en cuestiones como el fortalecimiento de la sociedad civil y de los canales para la participación social, pertenecientes al nivel del sistema, y en los que la cooperación española no se ha mostrado tan activa hasta el momento.

En el plano sectorial, se consideran aspectos como la regulación sectorial, la coherencia de las políticas y estrategias sectoriales, o la coordinación efectiva entre las organizaciones e institu- ciones que pertenecen a un mismo sector, ya se trate de organizaciones nacionales o del exterior y, en particular, la comunidad de donantes10. La cooperación española está dando sus primeros pasos en la definición de estrategias sectoriales de carácter general que habrá que ajustar a las condiciones particulares del contexto organizativo y de política en cada país (en julio de 2002 se aprobó la estrategia de medio ambiente y en 2003 está previsto elaborar y aprobar las de promoción de la democracia y Estado de De recho). Por su parte, el CAD de la O CDE recomienda a España estudiar la adopción de enfoques sectoriales, en colaboración con otros donantes, como vía para impul sar la apropiación y la sostenibilidad (M inisterio de Asuntos Exteriores, 2002, pág. 10).

En el nivel del sistema cabe considerar aspectos como las políticas, el marco jurídico, las normas, la coordinación institucional, las actitudes, los valores, el grado de conflicto social, etc., que configuran el contexto, y que pueden facilitar u obstaculizar el proceso de desarrollo.

Aquí la cooperación técnica española ha venido también de sempeñando al gunas labores de asistencia de manera regular, sobre todo en materia de asesoramiento para la modernización del marco jurídico en determinados sectores en el área iberoamericana, aprovechando la similitud de los sistemas administrativos y jurídicos de esta región con los españoles, cuestión que se suele interpretar, junto con la experiencia reciente de nuestro país, con una ventaja comparativa de España en relación con otros donantes.

La distinción entre los niveles citados nos lleva a la conclusión de que el cambio organizativo y el cambio institucional son cosas diferentes y que probablemente el primero sea una condición necesaria para el segundo, pero a menudo insuficiente. Asimismo, nos obliga a reconocer que es mucho más complejo y conflictivo incidir sobre el cambio institucional, sobre las reglas del juego, terreno en el que existe un complicado entramado de intereses políticos y sociales - y se puede llegar a cuestionar incluso el propio sentido o la legitimidad de algunas de las organizaciones con las que se trabaja- , que sobre el cambio organizativo, más manejable por estar circunscrito básicamente a aspectos de reforma organizativa 0 administrativa (D OVE, ¿?).

Asimismo, como se puede constatar, este nivel incluye aspectos mucho más complejos y un tanto etéreos, cuya apreciación resulta mucho más dificultosa a la hora de planificar intervenciones de desarrollo, establecer objetivos y fijar indicadores.

\section{3. ¿Q ué capacidades fortalecer en cada uno de los niveles?}

Una vez considerados los distintos planos del refuerzo institucional, habría que analizar cuáles son las áreas de capacidades más clásicas en las que las intervenciones de CT en este ámbito pueden incidir en cada uno de ellos. 


\section{Tabla 1}

Aspectios principalesa analizar en los cuatro planos dé refuerzo institucional

\begin{tabular}{|c|c|c|c|}
\hline Plano individual & Plano organizacional & Plano sectorial & Plano institucional \\
\hline $\begin{array}{l}\text { - H abilidades técnicas, profesionales } \\
\text { y/o académicas. } \\
\text { - H abilidades directivas } \\
\text { (planificación y gestión). } \\
\text { - Trabajo en equipo. } \\
\text { - M otivación, actitud, autonomía, } \\
\text { oportunidades de promoción, etc. } \\
\text { - Identificación con la organización. }\end{array}$ & $\begin{array}{l}\text { - Planificación estratégica (misión, } \\
\text { objetivos, etc.). } \\
\text { - Estructura organizativa y funcional } \\
\text { - Gestión financiera y } \\
\text { presupuestaria. } \\
\text { - Procedimientos técnicosy } \\
\text { administrativos. } \\
\text { - Gestión de recursos humanos } \\
\text { (selección, formación, incentivos, } \\
\text { etc.). } \\
\text { - Igualdad de género } \\
\text { - Sistemas tecnológicos y de } \\
\text { información. } \\
\text { - Relación con los ciudadanos } \\
\text { (prestación de servicios, servicios } \\
\text { de atención al clienteusuario) y } \\
\text { otras organizaciones } \\
\text { - Comunicación. }\end{array}$ & $\begin{array}{l}\text { - M arco jurídico-regulación } \\
\text { sectorial. } \\
\text { - Política sectorial. } \\
\text { - Legitimidad de las organizaciones } \\
\text { - Relaciones entreorganizaciones } \\
\text { (coordinación, complementariedad, } \\
\text { competencia, etc.). } \\
\text { - Privatizaciones/nacionalizaciones } \\
\text { - Cobertura y calidad en la } \\
\text { prestación de servicios. }\end{array}$ & $\begin{array}{l}\text { - M arco jurídico-normativo. } \\
\text { - Estado de D erecho. } \\
\text { - Contexto político, forma de } \\
\text { gobierno, relaciones de poder y } \\
\text { toma de decisiones. } \\
\text { - Políticas públicas y Administración } \\
\text { Pública. } \\
\text { - Legitimidad de las organizaciones } \\
\text { (corrupción, clientelismo, etc.). } \\
\text { - Sistema económico (contexto y } \\
\text { política macroeconómica, deuda, } \\
\text { mercado, competencia, protección } \\
\text { de las inversiones, etc.). } \\
\text { - Valores, creencias e ideales } \\
\text { culturales y religiosos. } \\
\text { - Participación social y canales para } \\
\text { ejercerla. }\end{array}$ \\
\hline
\end{tabular}

Sin ánimo exhaustivo, la tabla 1 resume algunas de los aspectos más relacionados con la capacidad en los cuatro planos citados. D e cual quier manera, la literatura sobre el tema es muy amplia y será el estudio de cada contexto y la preparación de cada intervención los que determinen en cada caso cuál o cuáles son los puntos de entrada dónde se ha de poner el énfasis.

\subsection{Algunas implicaciones procedimentales para la Cooperación Técnica Española}

La primera consecuencia que se deriva de este análisis para CT española es admitir que aunque la complejidad de las intervenciones aumente gradual mente conforme se transita desde el nivel individual, al organizativo, al sectorial y al del sistema, la creación de capacidad y refuerzo institucional son conceptos que, aunque muy relacionados con el tradicional enfoque de la formación de recursos humanos, necesariamente abarcan dimensiones más amplias.

La segunda es admitir que la definición de una estrategia de cooperación para el refuerzo institucional y el buen gobierno no puede venir marcada por el sumatorio de actividades ejecutadas en los sectores convencionalmente asignados a esta línea programática (desarrollo judicial, derechos humanos, procesos electorales, administración pública, etc.), sino que precisa la definición de un marco teórico en el que: a) Se plantee de manera explícita (y cuando sea posible, verificable) qué se pretende lograr con el fortalecimiento institucional en cada sector (definir las capacidades que se pretenden desarrollar 0 alcanzar)

b) Se defina, en cada caso y en cada momento, en qué nivel o niveles deben concentrarse los esfuerzos de desarrollo institucional, teniendo en cuenta que la incidencia exclusiva en uno de ellos tendrá por lo general efectos muy limitados sobre los otros y, por tanto, sobre la resolución real de los problemas institucionales.

c) Se establezca cuál es la conjunción apropiada de medios 0 estrategias para conseguirlo.

En el contexto de la cooperación española, la definición de una estrategia de refuerzo institucional debe llevar aparejada la introducción o consolidación, en su caso, de buenas prácticasl1 para la CT, que den respuesta a las principales carencias12 que enfrentan las intervenciones de refuerzo institucional. Entre esas buenas prácticas cabe destacar:

- La incorporación de las actividades clásicas - y a menudo aisladas- de la CT, tales como los cursos y seminarios, asistencias técnicas y consultorías, pasantías, suministro de equipos, etc., en programas y proyectos más amplios dirigidos al desarrollo de capacidades.

- Dado que el impulso para el refuerzo institucional no puede venir exclusivamente del exterior, en la selección de 
Tabla?

Problemas de falta de capacidad y estraterias resul tiantes

\begin{tabular}{|c|c|}
\hline Falta de capacidad causada por: & Estrategias de CT centradas principalmente en: \\
\hline Carencia de formación y habilidades técnicas. & Formación de recursos humanos en sus diversas modalidades, asistencia técnica. \\
\hline Ausencia de recursos. & $\begin{array}{l}\text { Infraestructura, equipamiento, pago de salarios, cobertura de gastos operativos, } \\
\text { apoyo presupuestario, etc. }\end{array}$ \\
\hline D eficiencias procedimentales. & Adecuación, rediseño y simplificación de procedimientos. \\
\hline D eficiencias organizativas. & $\begin{array}{l}\text { N uevas formas de organización, manuales de funciones, mecanismos de toma de } \\
\text { decisiones. }\end{array}$ \\
\hline Débiles sistemas de planificación y gestión. & Formación y asistencia técnica en materia de planificación y gestión. \\
\hline Bajos salarios y fuga de personal cualificado. & N uevos procedimientos laborales y de contratación, incentivos, etc. \\
\hline D ébil liderazgo en las organizaciones. & D esarrollo del liderazgo. \\
\hline $\begin{array}{l}\text { Escasez de organizaciones especial izadas para llevar a cabo determinadas } \\
\text { funciones o prestar determinados servicios. }\end{array}$ & C reación de nuevas organizaciones en determinadas áreas. \\
\hline Deficiente coordinación y colaboración entre organizaciones. & $\begin{array}{l}\text { Clarificación de la división del trabajo, búsqueda de complementariedades, } \\
\text { mejora de los sistemas de información, promoción del diálogo social. }\end{array}$ \\
\hline
\end{tabular}

entidades contraparte habrá de tenerse muy en cuenta en qué organizaciones existe una voluntad real de cambio.

- La realización de identificaciones mucho más rigurosas de las intervenciones de refuerzo institucional. Ello implica, en primer lugar, la realización de diagnósticos adecuados para cada uno de planos descritos y sus articula ciones, con el fin de obtener una valoración integral de la situación y la capacidad institucional (estructuras políticas y jurídicas, mapa institucional y de implicados, distribución y relaciones de poder, incentivos y resistencia al cambio, capacidad de gestión local y de absorción y re tención de la CT, estructura organizativa, procedimientos administrativos, habilidades, redes de información, etc.), así como identificar los problemas que se pretenden resolver con las intervenciones de refuerzo institucional13. En todo caso, la elaboración de esos diagnósticos no puede ser abordada desde una perspectiva meramente académica, sino que tienen que estar enfocados hacia la acción en el contexto correspondiente. Por otro lado, los diagnósticos no deberán realizarse de manera definitiva y cerrada antes de cada intervención, sino que a lo largo de la propia ejecución habrá que ir identificando y ayudando a enfrentar nuevos problemas de forma reflexiva.

Los medios o respuestas más idóneos para enfrentar los problemas en el seno de cada organización, sector o siste- ma más amplio se seleccionarán en función de la interpretación de la naturaleza de estos problemas y de sus causas y, por ende, de los objetivos que a raíz de esa interpretación se establezcan.

La tabla 2 ilustra con al gunos ejemplos cómo en función de los problemas que se detecten en los distintos niveles, podrían identificarse diversas estrategias de actuación para la CT. D ado que las causas de la débil capacidad no pueden atribuirse exclusivamente a la ausencia de conocimientos y habilidades técnicas individuales, la respuesta a los problemas de capacidad no debe concentrarse, exclusivamente, en la impartición de paquetes formativos 0 de asistencia técnica más o menos estandarizados. Por esta razón, las estrategias de la CT no siempre tendrán necesariamente que coincidir con enfoques intensiva 0 exclusivamente dedicados a la formación de recursos humanos.

H ace ya algún tiempo que se viene aceptando (todavía más en los discursos que en la práctica cotidiana de la cooperación) que la participación en cualquier tipo de proyecto desde sus inicios es una condición necesaria para la generación de compromiso, la apropiación del mismo por parte de los beneficiarios y otros agentes implicados y la propia viabilidad. En los proyectos de refuerzo institucional, además, esa participación es en sí misma un elemento 
central de la propia construcción de capacidades. Por esta razón, la recogida de información y elaboración del diagnóstico de partida ha de realizarse con los principales agentes y organizaciones implicadas, de forma que la propia capacidad para la recogida y análisis de la información se vaya creando en el país. Es imperativo ir reduciendo el a veces excesivo protagonismo de los donantes en materia de identificación y diseño de las propuestas de cooperación técnica y avanzar hacia fórmulas de asociación en la gestión de los programas de refuerzo institucional, eincluso en el propio manejo y control de los recursos.

- Para la selección de estrategias de intervención y el esta blecimiento de prioridades, no sólo habrán de tenerse en cuenta las posibles ventajas comparativas españolas sino, también y principalmente, las prioridades marcadas por la agenda de los países y organizaciones receptoras y por su propio compromiso de cambio institucional. Se trata en definitiva de partir dela situación real del socio con el que se trabaja, más que de la experiencia propia de cómo funcionan las cosas en el país del que los expertos proceden para su traslación e implantación en el nuevo contexto. Como bien explica BRIn Kerh off (1995, página 1), hay que tener en cuenta que no siempre es válida la mejor solución técnica vista desde la perspectiva del donante, sino la segunda o tercera mejor que cuente con el consenso y el respaldo adecuados.

La consideración de todos estos aspectos obliga a invertir más tiempo y mayores recursos antes de emprender la ejecución de las intervenciones de refuerzo institucional y durante la misma, e implica un cambio en el estilo con el que se viene realizando la CT, pero sin duda sienta bases más sólidas de compromiso mutuo y trabajo conjunto.

- Se admite generalmente que la complejidad de los proce sos de refuerzo institucional y desarrollo de capacidades precisa de una programación necesariamente flexible e iterativa y que en muchos casos no es posible obtener re sultados a corto plazo. N o obstante, es imprescindible dotar a las iniciativas de refuerzo institucional de una dirección clara, pasando de un modelo de CT que podríamos denominar «activista», caracterizado por la estandarización de los medios y la excesiva flexibilidad en los resultados, a otro que ponga el acento en los resultados en términos de capacidades, con flexibilidad en los medios y una apuesta decidida por el aprendizaje durante el proceso. Lo anterior supone, de alguna manera, combinar un enfoque de proceso durante la ejecución de las intervenciones de refuerzo institucional con la adopción, como requisito fundamental para la transparencia y el aprendizaje, de enfoques basados en el logro de objetivos 14 en dichas intervenciones.
- La identificación, desarrollo y selección de indicadores para las intervenciones de refuerzo institucional, esto es, la implantación de un sistema de medición y verificación de la capacidad. Si bien es sencillo encontrar indicadores a nivel de las actividades o resultados más inmediatos alcanzados por una cierta intervención de refuerzo institucional, reviste mucha mayor dificultad asignar indicadores cuando los objetivos de esas intervenciones se conciben en términos de desarrollo de capacidades en los niveles más amplios.

Por esta razón, con frecuencia se recurre al número de cursos impartidos o de alumnos que recibieron formación bajo tal o cual programa de refuerzo institucional en un determinado periodo, como medida de desempeño en el área de refuerzo institucional: «España proporcionó asimismo un fondo fiduciario al PN UD para la reforma judicial en Centroamérica, que impartió 270 cursos de formación en 24 ciudades de 7 países» (M AE, 2002, página 29). No obstante, eso no nos da ninguna medida de si esos programas formativos transmitieron capacidades, cuáles fueron esas capacidades y si éstas fueron recibidas, retenidas y aplicadas por los destinatarios de los mismos.

No es ésta una tarea exenta de dificultades. Para empezar, ya se ha indicado, la capacidad es un concepto muy vago y elástico, por lo que para poder asignar indicadores realmente operativos necesitamos una respuesta más clara a la cuestión de cuáles son las habilidades y funciones críticas (ya se trate de aptitudes y actitudes individuales, de funciones operativas de gestión o procedimentales o de funciones de carácter más estratégico) que se pretenden alcanzar en cada uno de los niveles (M ORGAN, 1997, página 5).

Por otra parte, como ya se ha indicado, se reconoce que los proyectos de refuerzo institucional incorporan un componente claro de proceso, de cambio en los comportamientos, de adaptación y aprendizaje que a veces puede chocar con un enfoque rígido de planificación por objetivos.

Asimismo, sería necesario avanzar en la investigación y selección de indicadores que permitan medir la influencia de los factores del entorno sobre los proyectos de re fuerzo institucional, así como en qué medida estos últimos generan impactos en el nivel del sistema.

- En todas las fases de las intervenciones de refuerzo institucional en las que intervengan expertos españoles, han de producirse asimismo cambios en el papel que éstos han venido desempeñando tradicionalmente y en el modo en que se organiza su participación en las iniciativas en este ámbito. En este sentido cabe señalar lo siguiente:

- En la medida en que las intervenciones de refuerzo institucional reúnen una dimensión técnica y política, 
el personal involucrado en las labores de CT debería combinar el perfil técnico que exige la transmisión de conocimientos y habilidades técnicas en un determinado sector (desarrollo judicial, tributación, fortalecimiento municipal, etc.), con el desempeño de un rol facilitador (más que conductor) del proceso. Una de las críticas a la asistencia técnica tradicional a través de expertos extranjeros se centra en que éstos, al suplir con su expertizaje las carencias de capacidad en un determinado contexto, suelen acentuar (más que contribuir a paliar) las debilidades institucionales 0 de capacidad. De ahí que se demande un «viraje hacia la facilitación» en la forma de prestar asistencia técnica16.

- H abrán de combinarse también la transmisión de conocimientos y habilidades en el sector correspondiente con el desarrollo y transmisión de herramientas y técnicas más propias de las intervenciones de refuerzo institucional (diagnóstico participativo del contexto y la organización, planificación estratégica, valoración de la sostenibilidad institucional, técnicas de negociación y resolución de conflictos, etc.).

- En relación con lo anterior, cada vez más se aboga por la formación de equipos multidisciplinares, que combinen la especialización técnica en el sector correspondiente con un perfil analítico, de planificación y de comunicación, en el que se tengan en cuenta otro tipo de habilidades como la paciencia con el ritmo lento del cambio institucional, la capacidad para facilitar el debate, el encuentro y la creación de relaciones de aso- ciación en el país, etc. Idealmente, por su conocimiento del contexto, su facilidad de interlocución y su influencia en ocasiones para implantar los cambios, es del todo conveniente contar con la participación de personal local en dichos equipos.

- La utilización de talleres participativos, como herramienta básica para la construcción de consenso, para la apertura de canales de participación y para la apropiación de las estrategias de refuerzo institucional por parte de los principales agentes implicados'16.

- En ocasiones convendrá complementar las modalidades más habituales de formación de recursos humanos. Así, a la formación en España podrían sumarse otras iniciativas de capacitación en el propio país y en el puesto de trabajo, pasantías en terceros países con un nivel de desarrollo similar, etc., medidas que aunque pueden reducir la visibilidad y el protagonismo del donante pueden tener mayor incidencia en el fortalecimiento institucional y el desarrollo de capacidades.

- Por último, con el fin de fomentar la sistematización de experiencias y el aprendizaje derivados de los programas de refuerzo institucional, debe promoverse en las instancias de cooperación española y, particularmente en la $\mathrm{AECl}$, el establecimiento de instrumentos como la creación de publicaciones especializadas, la realización de proyectos piloto, el intercambio regular de información, la participación en redes, foros y grupos de discusión, la realización de evaluaciones en este campo y la difusión regular de las enseñanzas obtenidas.
* Luis Cámara (Icamara@wanadoo.es) es economista, postgraduado en planificación y políticas públicas. Es miembro de Acciones de D esarrollo y Cooperación (ADC) y del Consejo Asesor de CIDEAL. H a sido director del Centro Iberoamericano de Formación de la Agencia Española de Cooperación Internacional (AECI) en Cartagena de Indias (Colombia) y del Centro Europeo para la Formación de Estadísticos de Países en D esarrollo (CESD -M adrid). En la actualidad desarrolla labores de docencia especializada y asistencia técnica para la gestión de programas y proyectos de cooperación. El autor agradece los provechosos comentarios al texto de Rafael C ascante, H ugo C amacho, H éctor Sainz y M anuel Gómez Galán.

1 El Comité de Ayuda al desarrollo (CAD) de la O rganización de Cooperación y Desarrollo Económico (OCDE) proporciona una definición amplia de $\mathrm{CT}$, que comprende «las actividades financiadas por un país donante cuyo propósito principal es aumentar el nivel de conocimientos, habilidades, saber hacer técnico 0 actitudes productivas de los habitantes de los países en desarrollo» (D AC, 2000, página 12). Por su parte, la Ley 23/1998, en su artículo 10 define la CT en parecidos términos y explica que la misma se articula mediante programas y proyectos de refuerzo de la formación y de asesoramiento técnico, aportación de estudios o transferencia de tecnologías. En Agencia Española de C ooperación Internacional (2002, página i ), se especifica que « os principales instrumentos de la CT son aquellos relacionados con la formación de recursos humanos y la asistencia técnica, esto es, las acciones formativas regladas o informales, las prestaciones de expertos y labores de consultoría y el asesoramiento técnico y las pasantías».
2 El Banco M undial (1996) estima que la asistencia técnica que ha venido proporcionando ha sido más exitosa en la consecución de determinados productos concretos (por ejemplo, de preparación de proyectos de inversión) que en términos de desarroIlo institucional. De un total de 1.689 proyectos con fines de desarrollo institucional aprobados en el periodo 1971-91, únicamente un 29\% tuvo un impacto significativo sobre el desarrollo institucional; en un $45 \%$ de los casos el impacto fue modesto y en un 26\% fue insignificante. World Bank (1996).

3 Por ejemplo, transparencia y control de legalidad contra oportunidad en la toma de decisiones.

4 En raras ocasiones los programas y proyectos cuyos objetivos hacen referencia al re fuerzo o desarrollo institucional, llevan aparejados indicadores operacionales que permitan verificar en qué medida los proyectos contribuyen a ese desarrollo institucional. Una aproximación al tema de indicadores del desarrollo de la capacidad puede encontrarse en M ORGAN (1997) Una propuesta muy amplia y desarrollada de indicadores de gobernabilidad, específicamente adaptada a la estrategia de los EEUU en este campo, puede encontrarse en USAID (1998).

5 D ove (¿?, página 2) hace una descripción muy ilustrativa de la clásica diferencia conceptual entre instituciones y organizaciones, y de las implicaciones que esa diferencia tiene para abordar un proceso de cambio institucional.

6 BOLGER (2000, página 5). Incorpora el nivel «sectorial o de la red», justificando su inclusión por la especial atención que en la actualidad tienen los enfoques sectoriales. N ORAD (2000) excluye el plano sectorial, contemplando únicamente los otros tres 
niveles. N ótese que serán las circunstancias particulares de cada contexto las que aconsejen el análisis más apropiado, pudiéndose complicar o simplificar el esquema propuesto..

${ }^{7} \mathrm{~A}$ juicio del autor, la cooperación para el desarrollo debería abordar con mucho mayor énfasis el apoyo a la implantación de una carrera profesional en la administración pública de los países en desarrollo, con el fin de que la inversión en la formación de empleados públicos de esos países adquiera pleno sentido y aumente su eficacia en términos de refuerzo institucional. La permanencia del personal en la administración pública no sólo afecta a la profesionalización del mismo, sino que es también una garantía para los ciudadanos de la aplicación de la normativa.

8 La CT española viene trabajando también en este ámbito, sobre todo en materias relacionadas con la reforma y modernización del sector público, con la descentralización y el fortalecimiento municipal, la modernización de la administración tributaria, Fuerzas de Policía, etc., en los países en desarrollo, si bien el enfoque adoptado en una buena parte de los casos ha seguido gravitando básicamente sobre el primer nivel, centrándose de forma abrumadoramente mayoritaria en la formación de recursos humanos.

9 La continua prestación deficiente de servicios por parte del Estado genera desmotivación y desinterés en los ciudadanos para ejercer la presión social en favor de un mejor servicio; a su vez, la escasa presión de la ciudadanía desincentiva al Estado, permitiéndole seguir prestando deficientemente esos servicios.

10 Una aproximación al tema de los enfoques sectoriales (Sector W ide Approaches 0 SWAP) puede obtenerse en FOST ER (2000).

11 Algunas 0 ficinas Técnicas de Cooperación de la AECI están avanzando progresivamente en el proceso de reflexión y puesta en marcha de buenas prácticas en la identificación, diseño y ejecución de intervenciones de refuerzo institucional (N icaragua es un ejemplo ilustrativo al respecto), pero en otras la situación es todavía muy débil.
$12 \mathrm{El}$ Banco M undial (2000), en un informe sobre sus intervenciones de cooperación en materia de desarrollo institucional, señala que las causas principales de los fracasos son: - Condiciones desfavorables en la gobernabilidad y el contexto institucional.

- Falta de apropiación, participación y compromiso de los prestatarios.

- Ausencia de una estrategia de desarrollo institucional, pobres diagnósticos del contexto institucional y escasa utilización de las capacidades ya existentes.

- D ébil capacidad de gestión de los prestatarios, incluyendo carencia de personal y habilidades para facilitar el seguimiento y la evaluación del desarrollo institucional.

13 Un esfuerzo reseñable es el Diagnóstico Institucional de la República del Paraguay, recientemente elaborado por el Instituto Internacional de Gobernabilidad de C ataluña por encargo del PN UD (obtenible en http://www.iigov.org/numerol00/).

14 EI CAD de la OCDE recomienda se afine el Plan Director de la Cooperación Española para que refleje una jerarquía más clara de principios y objetivos, así como la adopción de un enfoque más orientado a los resultados en la programación y ejecución, con el fin de extraer las lecciones pertinentes (M AE, 2002, pág. 7).

Algunos ejemplos concretos de aplicación práctica del enfoque de planificación por objetivos a programas de refuerzo institucional puede consultarse en CÁm ARA, L./ Cervera, J.L y Sainz, H (2001): Aplicación del Enfoque del M arco Lógico a la cooperación internacional en materia estadística y C AM ACH O, H ugo; CÁm ARA, Luis; Cascante, Rafael y Sáinz, H éctor (2001): El Enfoque del M arco Lógico: 10 casos prácticos.

15 Utilizando la terminología de BRIN KERH OFF (1995, página 4), el estilo de prestar asistencia técnica debe transitar progresivamente por la secuencia: experto «hacedor» - experto consultor- experto formador-facilitador/socio/catalizador.

16 Sobre las ventajas de la utilización de talleres participativos puede consultarse Brinkerhoff (1994). Una reflexión sobre su organización y aplicación en la práctica se puede obtener en Camacho y otros (2001).

\section{Referencias bibliográficas}

Agencia Española de Cooperación Internacional (2000): Ley de Cooperación Internacional para el desarrollo y disposiciones reglamentarias. Vicesecretaría G eneral. Centro de Información. Marzo.

Agencia Española de Cooperación Internacional (2002): Catálogo de cooperación técnica española. D ocumento de Trabajo para uso interno, Febrero.

BASER, H ./ BOLgER, J. (1996): From Technical Cooperation to Capacity development: Changing Perspectives in CIDA.CIDA Policy Branch, February. 0 btenible en http://remote4.acdi-cida.gc.ca/extranet/policy/cdbboard.nsf/56fa583b4ea5589d $85256793005318 \mathrm{bf} / 4$ eldg2fb20085f32852569da0018d091/\$FILE/199602\% 20From\% 20TC\% 20to\% 20CD.doc.

Bolger, J. (2000): Capacity development: Why, What and How. CIDA, Policy branch. 0 ccasional Series. Vol.1 No 1, M ay. O btenible en http://remote4.acdicida.gc.ca/extranet/policy/cdbboard.ns/56fa583b4ea5589d85256793005318bf/d0de caf5bef4387b852569da00097236/\$FILE/CapD evOSVol1N 01-E.pdf.

BRIN KERH OFF, D erick W. (1994): Using workshops for Strategic M anagement of Policy Reform. U SAID 's Implementing Policy Change Project.Technical N ote N 06 , US Agency for International Development, June. Washington DC. Obtenible en http://www. acdi-cida.gc.ca/IN ET//M AGES.N SF/vLU Image//C apacityD evelopment/ \$file/Brinkerhoff-UsingWorkshops-E.pdf.

BRIN KERH OFF, D erick W. (1995): «Technical Cooperation for Capacity-Building in Strategic Policy M anagement in D eveloping C ountriess. D ocumento presentado en la 56 a Conferencia Nacional de la American Society for Public Administration. San Antonio TX, July 22-26. US Agency for International Development. 0 btenible en (www.capacity.org/pubs/conf/brinkerhoff-total.htm).

Camacho, Hugo; Cám ara, Luis; Cascante, Rafael y Sainz, Héctor (2001): El Enfoque del M arco Lógico: 10 casos prácticos. ADC-CIDEAL, Madrid.
Cám ara, L./ Cervera, J.L y Sainz, H (2001): «Aplicación del Enfoque del Marco Lógico a la cooperación internacional en materia estadística». Revista Española de D esarrollo y Cooperación, n- 9 otoño/invierno (Paginas 137-159). M adrid.

Comité de Ayuda Al D esarrollo (2002): G losario de los principales términos sobre evaluación y gestión basada en resultados. Grupo de trabajo del CAD sobre evaluación de la Ayuda. Evaluation Aid and Effectiveness n- 6. O rganización de Cooperación y D esarrollo Económico. París. O btenible en http://www. oecd.org/pdf/ M $00030000 /$ M 00030075.pdf.

Development Assistan Ce Committee (1996): Synthesis report from the D AC Informal network on technical co-operation. O rganisation for Economic Cooperation and D evelopment, París (obtenible en http://www.oecd.org/dac).

Developm ent Assistan Ce Committee (1997): Final report from the DAC Informal network on technical co-operation of the ad hoc Working Group on Participatory D evelopment and Good Governance. O rganisation for Economic C 0-operation and D eve lopment, París (obtenible en http://www. oecd.org/dac).

Development Assistance Committee (2000): D AC Statisical Reporting Directives. O rganisation for Economic C 0-operation and D evelopment. M ay, Paris. Disponible en http://www1.oecd.org/dac/htm/dacdir.htm\#K ey\%20D efinitions.

D OVE, S. (i?): «Las decisiones políticas: Instituciones v/s organizaciones». Paper no 7. Colección de Papers. Instituto Internacional de Gobernabilidad de Cataluña, BarceIona. Disponible en http://iigov.org/papers/tema4/paper0007.htm.

FOSTER, M . (2000): «N ew Approachesfor D evelopment Co-operation: What can we learn from experience with implementing Sector W ide Approaches?». Working Paper 140. Centre for Aid and Public Expenditure. O verseas D evelopment Institute. London. 0 btenible en http://www.odi.org.uk/publications/wp140.pdf. 
Instituto Internacional de Gobernabilidad de Cataluña (2002): Diagnóstico institucional de la República de Paraguay. ( 0 btenible en http://www.iigov.org/numero100/).

JACOBS, C. (1998): «nstitutional strengthening and technical co-operating: developing a best practice model». Journal of International D evelopment, 10, pag. 397-406, 0 ctober 1998) http://www. euforic. org/rondzend/nov981.htm.

LAND, A. (2000): Implementing Institutional and Capacity D evelopment: Conceptual and O perational Issues (ECDPM Discussion Paper 14) M aastrich: European Centre for D evelopment Policy M anagement. (O btenible en http://www.oneworld.org/ecdpm/ pubs/dp14_gb.htm).

Lusthaus, Charles/ Gray, David/ Adrien, M arieH élène (1996): «trengthening Institutions in the D eveloping World: Trends and Issues». U niversalia 0 ccasional Paper $\mathrm{N}$ 18, N ovember. Universalia. M ontreal (O btenible en http://www.universalia. com/files/occas18.pdf).

Lusthaus, Charles/ Adrien, M arie-Hélène/Perstinger, Mark (1999): «C apacity D evelopment: definitions, Issues and Implications for Planning, M onitoring, and Evaluation». U niversalia 0 ccasional Paper, $N 035$, September. U niversal ia. M ontreal. 0 btenible en http://www. universalia.com/files/occas35.pdf.

M ENTZ, J.C.N . (1997): «Personal and institutional Factors in Capacity Building and Institutional Development». (ECD M Working Paper N 014$)$ : M aastricht: European Centre for Development Policy Management http://www.oneworld.orglecdpm/ pubs/wp14_gb.htm.

M Inisterio de Asuntos Exteriores (2002): Informe del CAD sobrela Cooperación Española para el desarrollo. N oticias y D ocumentación de la Cooperación. Oficina de Interpretación de Lenguas. (0 btenible en www. aeci.es).

M organ, P. (1997): «The design and use of capacity development indicators». Paper prepared for the Policy Branch of CIDA, D ecember. Canadian International Development Agency (CID A). 0 btenible en http://remote4.acdi-cida.gc. ca/extranet/policy/cdbboard.ns/56fa583b4ea5589d85256793005318bf/c97 c93c328a8d490852569 da000ed313/\$FILE/1997-12\% 20M organ\% 20-\% 20indicators\% 20paper\%20\%20 (W kshp\% 2011).doc.

Morgan, P. (1998): Capacity and Capacity Development-Some Strategies. Policy Branch, , Political and Social Policies Division, Canadian International development Agency. 0 ctober. Obtenible en http://www.acdicida.gc.ca// N ET //M AGES.N SF/VLU Images/CapacityD evelopment/\$file/1998-10paper(Wrkshp11).PD F.

N ORAD (2000): Handbook in Assessment of Institutional Sustainability, 0 slo, June. N orwegian Agency for D evelopment C ooperation.

OCDE (1996): DAC Seminar on Technical Co-operation and Capacity development: Synthesis report. D evelopment C 0-operation D irectorate. $M$ arzo, $O$ rganisation for Economic C 0-operation and D evelopment. O btenible en http://www1.oecd.org/dad TCN ET/pdf/tc_sem96e.pdf.

OverSeas D evelo pm ent Ad ministration (1992): The process approach to projects. Londres, multicopiado.
PN UD (1997): D esarrollo de la capacidad. M onografía de asesoramiento técnico № 2. D ivisión de D esarrollo de la gestión y de gobernabilidad. Dirección de Política de D esarrollo. Programa de naciones U nidas para el D esarrollo. N ueva York http:// magnet. undp.org/D ocs/cap/Capdevsp.pdf.

Q UALM AN, A./ M ORGAN, P. (1996): Applying results-based management to capacity de velopment. Political and Social Dimensions Division. Policy Branch, CIDA, Fe bruary.. 0 btenible en http://remote4.acdi-cida.gc.ca/extranet/policy/cdbboard.ns/56fa 583b4ea5589d85256793005318bf/e39ba7ed6a07b02285256a16007ela7c/\$FILE/1 996-02\% 20Q ualman\%20and\% 20M organ\%20CD\%20and\% 20RBM.pdf.

RondinelLI, D ennis A. (1993): D evelopment projects as policy experiments: An adaptative approach to development admini stration, Routledge, Londres-N ueva York.

Schacter, M . (2000): «"Capacity building": A N ew Way of D oing Business for De velopment Assistance 0 rganizations», Policy Brief, N 으. January. Institute O n Governance 0 ttawa, Canada. O btenible en http://iog.ca/publications/policybrief6. pdf.

SChaCter, M . (2001): «T he H eart of the M atter: D onors, D evelopment Assistance, and Public Sector Reform», Policy Brief, N 10. February. Institute O n Governance 0 ttawa, C anada. 0 btenible en http://iog.ca/publications/policybrief10.pdf.

Secretaría de Estado para la CoOperación Internacional y para IberiomériCA, SECIPI (2001): Plan Director de la Cooperación Española 2001-2004. M inisterio de Asuntos Exteriores. 0 btenible en http://www. aeci.es/4-Legisacion/ftp/PlanD irector.pdf.

SINGH, S. (2002): Technical cooperation and stakeholder ownership. United Nations D evelopment Programme. N ew D elhi. 0 btenible en http://capacity.undp.org/focus/ modalities/modalitiespdf.

USAID (1983): Institutional D evelopment. USAID Policy Paper. Bureau for Program and Policy coordination. US Agency for International Development. Washington, D C. 20523, M arch. 0 btenible en http://www. usaid.gov/pubs/ads/200/instdev/instdev. pdf.

USAID (1998): «H andbook of Democracy and Governance Program Indicators». Technical Publications Series, August. Center for D emocracy and Governance. Bure au for Global Programs, Field Support, and Research. U.S. Agency for International D evelopment. Washington, D.C. 20523-3100. 0 btenible en http://www. usaid. gov/democracy/pdfs/pnacc390.pdf.

W OrLd BAn K (1996): Technical Assistance. Lessons and Practices. Number 7. 0 pera tions Evaluation D epartment. World Bank, Washington DC. Una síntesis de las principales conclusiones y recomendaciones puede obtenerse en http://wbln0018. worldbank.org/oed/oeddoclib.nf/812f6434a83d93ba85256808006a0024/1369d06d5 2b3c791852567f5005d80ab?0 penD ocument.

W ORLD BANK (2000): TheWorld Bank experience with insitutional development. Lessons and Practices. N umber 14. O perations Evaluation D epartment. World Bank, Washington $D C$. Una síntesis de las principales conclusiones y recomendaciones puede obtenerse en http://wbln0018.worldbank.org/oed/oeddoclib.ns/812f6434a83d93ba 85256808006a0024/fd17b8f0ed4179d2852569ba006f34a4?O penD ocument. 